\title{
ODYSSEUS: DESCRIPTION OF AND RESULTS FROM A STRONG- -STRONG BEAM-BEAM SIMULATION FOR STORAGE RINGS
}

\author{
E. B. Anderson * , T. I. Banks, J. T. Rogers, LNS, Cornell University, Ithaca, NY \\ Abstract \\ ODYSSEUS is written in Fortran77 for portability.
}

ODYSSEUS is a simulation of the beam-beam interaction in $e^{+} e^{-}$storage ring colliders which is specifically intended to reveal the dynamic collective behavior of the colliding beams. This program is a true sixdimensional strong-strong simulation in which the electromagnetic fields of longitudinal slices of the colliding beams are recalculated for each slice collision. Broadband wake fields are included. No constraints are placed on the distribution of particles in the beams. The program achieves its speed through adaptively choosing between alternative electromagnetic field calculation methods. Benchmarking tests and various results will be presented.

\section{INTRODUCTION}

In storage ring colliders the beam-beam forces produce changes in the distribution of particles that can be important to the stability of the beams and the luminosity. Because the beam-beam force is strongly nonlinear, exact calculations are difficult and particle tracking simulations are more suitable. Strong-strong simulations, in which the force exerted on each beam by the opposing beam is calculated, are capable of generating self-consistent charge distributions and have been used to examine the collective behavior of round beams [1,2], charge-compensated round beams [3], and beams of arbitrary ellipticity [4]. These simulations are very time-intensive because of the need to repeatedly calculate the electromagnetic field of each beam, and previous unconstrained strong-strong simulations have therefore included only transverse degrees of freedom.

This paper presents a new beam-beam simulation program, ODYSSEUS (Optimized DYnamic Strong-Strong E-plus e-minUs Simulation). To the authors' knowledge, ODYSSEUS is the first six-dimensional strongstrong beam-beam simulation in which no constraints are placed on the beams and is the first to include wake fields. These features make it possible to investigate any mode of oscillation of the colliding beams, including head-tail modes.

ODYSSEUS is capable of rapidly calculating the electromagnetic field of a beam divided into many longitudinal slices because it adaptively chooses from a variety of different field computation methods. Different algorithms are used for the core, the transverse tails, and the longitudinal tails of the beam. The parameters of the program can be changed to model flat or round beams. Further, inclusion of the longitudinal degree of freedom and wake fields allows the investigation of previously inaccessible physics.

\footnotetext{
*Email:eba1@cornell.edu
}

\section{PARTICLE TRACKING}

\subsection{The Storage Ring}

On each simulated turn through the storage ring, each macroparticle is propagated from the collision point and back again through the linear optics of the storage ring, including chromaticity, synchrotron radiation excitation and damping, RF phase focusing, and wake field deflections. Macroparticles which have migrated past a transverse aperture are no longer considered in the simulation. Longitudinal and transverse short-range wake fields are included in the simulation. An input file contains a list of longitudinal and transverse resonators with values for the resonant frequency $\omega_{R}$, shunt impedance $R_{s}$, and quality factor $Q$ of each resonator.

\subsection{Collisions}

During its passage though the opposing bunch, the transverse position of each macroparticle may change appreciably because the vertical interaction point beta function, $\beta_{V}^{*}$, in CESR and many other colliders is comparable to the bunch length, $\sigma_{z}$. ODYSSEUS handles the longitudinal variation of the electromagnetic field of the beam by dividing the beam into slices. The simulation collides each pair of slices sequentially, updating the transverse momenta and positions of each macroparticle after each pairwise collision of slices. For each slice collision the slice electromagnetic field is calculated by one of the methods described below. Macroparticles undergoing longitudinal oscillations may migrate from slice to slice, so on each turn the macroparticles are sorted according to their longitudinal position and reassigned to slices.

\section{ELECTROMAGNETIC FIELD CALCULATION}

\subsection{Beam core}

If the number of macroparticles, $N$, within a slice is very small, the integrated field at a probe beam macroparticle is calculated from the exact radius vector from each opposing source beam macroparticle. The field must be calculated at the position of each macroparticle in the probe beam, so the number of calculations goes as $N^{2}$, making this method efficient only for $N<50$.

For larger values of $N$, the electromagnetic field is calculated on a rectangular grid using pre-calculated Green's functions for charges on the grid points. The macroparticle 


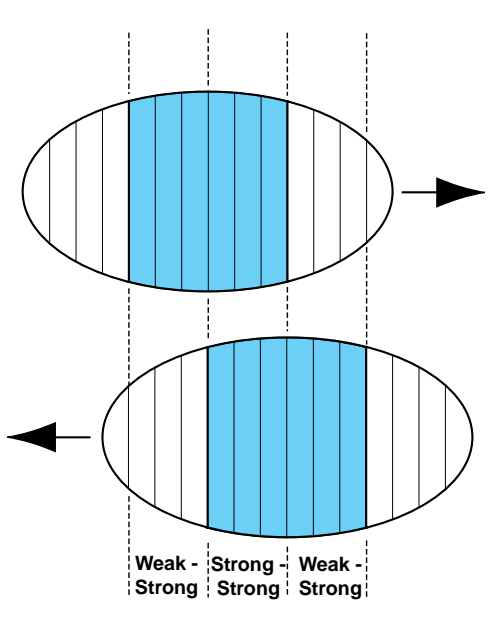

Figure 1: The beam is divided into longitudinal slices and the collision of each pair of slices is simulated in sequence. Slices are marked as either "weak" (white) or "strong" (gray) and the appropriate technique is applied when they interact. No two weak slices ever interact.

charge is assigned to the grid points using one of two areaweighted techniques described in below. If the number of grid points $N_{g}$ is less than about 200, the convolution of the charge density and Green's function is done as a summation in real space. The number of calculations required for this convolution goes as $N_{g}^{2}$.

For larger values of $N_{g}$, the convolution of the Green's functions and charge density is done as a simple multiplication in wavenumber space. The speed of this method is limited by the speed of the necessary Fourier transform to wavenumber space and the inverse transform back to real space. The number of calculations goes as $N_{g} \log _{2} N_{g}$. To suppress edge effect problems in the Fourier transforms, the size of the wavenumber space can be doubled in both directions and padded with zeros [5] although this is found to be unnecessary for a typical, Gaussian-like, charge distribution.

\subsection{Beam tails}

The tails of the beam, typically taken to be particles with a displacement of more than $(10 / 3) \sigma$ in any direction, are treated differently than the core particles. The tail particles have very little effect on the beam-beam force. They do, however, respond to the beam-beam force, so a weakstrong calculation is used.

Longitudinal tail particles are subject to forces from the core of the opposing beam. This is a weak-strong calculation. A full calculation of the field from the opposing (strong) beam slice is performed, but the tails are assumed to have no effect on the other beam. All slices may be treated as strong-strong slices if the user so chooses.

The transverse tail particles are subject to a beam-beam force of similar magnitude to that experienced by the core particles. The fine structure of the charge distribution of the core has little influence on the field in the transverse tails, so the core is assumed to be a two-dimensional Gaussian charge distribution with the same total charge and transverse moments as the slice. The field from this Gaussian charge distribution is calculated from the rational approximation of Talman and Okamoto [7].

\subsection{Interpolation techniques}

Whenever a grid-based technique is used, it is necessary to interpolate from the fields on a grid to arbitrary particle locations and from the particle locations to the grid when depositing charge. Odysseus gives the user a choice between two interpolation methods: a four-point CloudIn-Cell (CIC) technique and a nine-point extension of the Triangular-Shaped Cloud (TSC) technique[5]. Higher order interpolation schemes tend to blur the detail in the charge distribution. In ODYSSEUS a "sharpening function" is used during the convolution of the charge density and Green's function in wavenumber space [6].

\section{PRE- AND POST-PROCESSING}

A user interface, PENELOPE, has been written to produce the input files used by ODYSSEUS. Parameters may be read from existing files and modified, or may be entered through the keyboard. PENELOPE works with a set of accelerator parameters which is convenient for the user, but which is larger than the set of independent parameters. As new parameters are entered, derived parameters are calculated and displayed. Thus, self-consistency between parameters is enforced, unless the user specifically chooses otherwise.

ODYSSEUS was designed to investigate the coherent oscillations of the bunch, so post-processing to analyze the bunch spectrum is necessary. Post-processing is done in a Mathematica [8] notebook designed to look at beam-beam modes and their growth and damping rates.

\section{SPEED AND ACCURACY CONSIDERATIONS}

\subsection{Field error due to transverse grid}

For round beams a field calculation grid can be constructed out of square cells with equal numbers of cells in each dimension. This construction allows for accurate field calculation with low numbers of grid squares. In contrast, the often extreme aspect ratios of flat beams force the use of either large numbers of cells or individual cells with poor aspect ratios. When trying to model flat beams with a minimum number of grid cells, one may give the grid cells an aspect ratio other than unity, but there are dangers in this choice. If a potential is used to calculate the fields, then the choice of the value of the Green's function at the origin is important. ODYSSEUS uses a separate Green's function for each component of the electric field and the maximum aspect ratio usually allowed is 1.4. 


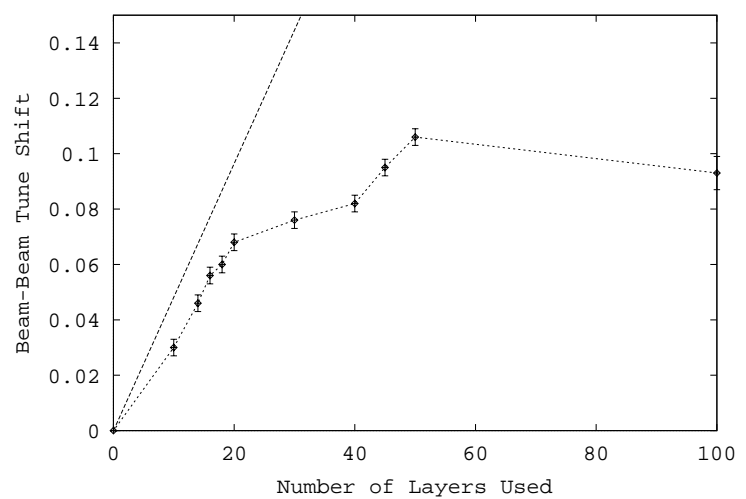

Figure 2: The figure above shows the vertical beam-beam tune shift, $\Delta \nu_{y}$, as a function of the number of slices. The straight line is an estimate of the maximum beam-beam parameter, $\xi_{y}$, from slicing errors alone (See Equation 1).

\subsection{Field error due to longitudinal slicing}

Previous investigators [9] had indicated that few slices, five or less, are necessary to model the colliding beams. We instead found that the error introduced by slicing increases the simulated emittance of the beam, so that the simulation produces a maximum value of $\xi$. This limit is severe when $\beta_{y}^{*}$ is so small that it is comparable to the bunch length $\sigma_{z}$. In a particle-tracking simulation using longitudinal slices of uniform width, particles at the front and back of each slice receive a different deflection than would actual beam particles. From this we find that a horizontal tune shift can be produced due to the finite slice length. We find this to be:

$$
\xi_{y, \max }=\frac{4 \sqrt{3}}{\pi} \frac{\beta_{y}^{*}}{\Delta z} \sqrt{\delta}
$$

Other physical or numerical effects may further reduce $\xi_{y}$. Fig. 2 shows the vertical beam-beam tune split as a function of the number of slices. The behavior supports the result of Eq. 1 that there is a maximum tune split that is inversely proportional to the slice length. Simulations of storage rings with a small natural vertical emittance require a large number of slices. Slices of non-uniform length [9] may reduce the effect.

\subsection{Speed}

ODYSSEUS is currently being run on a farm of $500 \mathrm{MHz}$ Alpha-chip personal computers running Linux. The fast Fourier transform takes almost $90 \%$ of the time taken by the code for a typical run. The speed of ODYSSEUS may be compared to that of a non-adaptive PIC-only calculation by running with PIC-only options and comparable parameters. The calculation takes five times longer than a calculation with the optimized code.

\section{CONCLUSIONS}

There are a number of computational advances represented in ODYSSEUS. Its adaptive nature allows it to run several times faster than a code using PIC methods alone. The Green's function grid used in the PIC calculations is pregenerated and recalculated as necessary. Approximations are used in the transverse and longitudinal tails in order to save time on calculations. ODYSSEUS incorporates wake fields, enabling the user to calculate collective instability thresholds for colliding beams. Runs can be done in reasonable amounts of time, ranging from an hour to a few days depending on the approximations used and beam shape.

Three unexpected results became evident during this work. The first is the necessity for grid aspect ratios close to $1: 1$ when using an electric field Green's function, and exactly $1: 1$ when using an electric potential Green's function. The second was the discovery of a minimum (simulated) emittance due to the nonzero longitudinal slice width. The third was that using zero-padding in the Fourier transform was not the most efficient means to minimize errors.

Three interesting results have already been found. The ratio between the beam-beam parameter and the coherent tune split has been calculated, the shift in head-tail mode frequencies for colliding beams have been investigated, and the effect of additional wiggler magnets on CESR's luminosity approximated.

\section{ACKNOWLEDGMENTS}

This work was supported by the National Science Foundation. The authors extend their thanks to R. Talman, G. Codner, J. Irwin, R. Siemann, M. Furman, S. Krishnagopal, R. Ryne, R. Ng, and R. Helmke.

\section{REFERENCES}

[1] S. Krishnagopal, Ph.D. Thesis, 1991, Cornell University.

[2] S. Krishnagopal and R. Siemann, Phys. Rev. Lett. 67 (1991) 2461.

[3] B. Podobedov and R.H. Siemann, Phys. Rev. E 52 (1995) 3066.

[4] S. Krishnagopal, Phys. Rev. Lett. 76 (1996) 235.

[5] R.W. Hockney and J.W. Eastwood, Computer Simulation Using Particles, Hilger, Bristol, U.K., 1988.

[6] E. B. Anderson, ODYSSEUS: A Dynamic Strong-Strong Beam-Beam Simulation for Storage Rings, PhD. Dissertation, Cornell University (1998); Available at http://www.ruph.cornell.edu/edwin/.

[7] Y. Okamoto and R. Talman, Cornell Laboratory of Nuclear Studies report CBN 80-13 (1980).

[8] Mathematica, Wolfram Research, Inc., Champaign, Illinois, USA.

[9] M. Furman, A. Zholents, T. Chen, and D. Shatilov, Lawrence Berkeley National Laboratory report PEP-II/AP Note 95.39/LBL-37680/CBP Note-152 (1995). 